\title{
CONF-950226--53
}

UCRL-JC-119257

PREPRINT

\section{Applications of Microlens-Conditioned Laser Diode Arrays}

\author{
Raymond J. Beach, Mark A. Emanuel, Barry L. Freitas, Jay A. Skidmore, \\ Nils W. Carlson, William J. Benett, and Richard W. Solarz
}

This paper was prepared for submittal to Laser Diode Optics II, SPIE Photonics West '95

San Jose, CA, February 4-10, 1995

\section{January 1995}

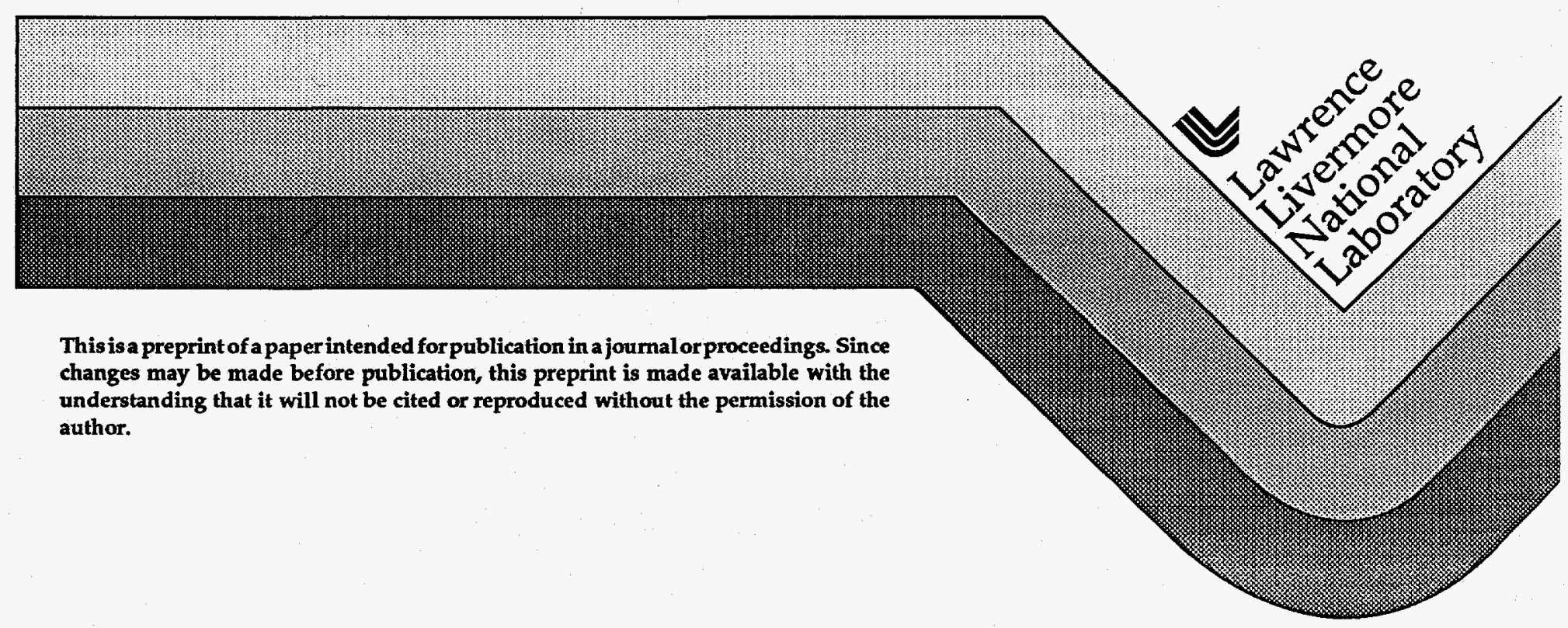

DISTRIBUTION OF THIS DOCUMENT IS UNLMIED 


\section{DISCLAIMER}

This document was prepared as an account of work sponsored by an agency of the United States Government. Neither the United States Government nor the University of Californiz nor any of their employees, makes any warranty, express or implied, or assumes any legal liability or responsibility for the accuracy, completeness, or usefulness of any information, apparatus, product, or process disclosed, or represents that its use would not infringe privately owned rights. Reference herein to any specific commercial products, process, or service by trade name, trademark, manufacturer, or otherwise, does not necessarily constitute or imply its endorsement, recommendation, or favoring by the United States Government or the University of California. The views and opinions of authors expressed herein do not necessarily state or reflect those of the United States Government or the University of California, and shall not be used for advertising or product endorsement purposes.

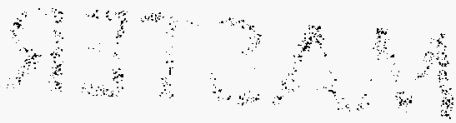




\section{DISCLAIMER}

Portions of this document may be illegible in electronic image products. Images are produced from the best available original document. 


\title{
Applications of Microlens-Conditioned Laser Diode Arrays
}

\author{
Raymond J. Beach, Mark A. Emanuel, Barry L. Freitas, Jay A. Skidmore, \\ Nils W. Carlson, William J. Benett, and Richard W. Solarz
}

\author{
Lawrence Livermore National Laboratory \\ P.O. Box 808, L-495, Livermore, CA 94550 \\ (510) 423-8986, (510) 422-3358 Fax
}

\begin{abstract}
The ability to condition the radiance of laser diodes using shaped-fiber cylindrical-microlens technology has dramatically increased the number of applications that can be practically engaged by diode laser arrays. Lawrence Livermore National Laboratory (LLNL) has actively pursued optical efficiency and engineering improvements in this technology in an effort to supply large radiance-conditioned laser diode array sources for its own internal programs. This effort has centered on the development of a modular integrated laser diode packaging technology with the goal of enabling the simple and flexible construction of high average power, high density, two-dimensional arrays with integrated cylindrical microlenses.

Within LLNL, the principal applications of microlens-conditioned laser diode arrays are as high intensity pump sources for diode pumped solid state lasers (DPSSLs). A simple end-pumping architecture has been developed and demonstrated that allows the radiation from microlens-conditioned, two-dimensional diode array apertures to be efficiently delivered to the end of rod lasers. This architecture enables the generation of pump beams that are scalable in absolute power with intensities approaching $100 \mathrm{~kW} / \mathrm{cm}^{2}$. To date, pump powers as high as $2.5 \mathrm{~kW}$ have been delivered to $3 \mathrm{~mm}$ diameter laser rods. Such high power levels are critical for pumping solid state lasers in which the terminal laser level is a Stark level lying in the ground state manifold. Previously, such systems have often required operation of the solid state gain medium at low temperature to freeze out the terminal laser Stark level population, so as to minimize loses resulting from reabsorption of the laser radiation. The necessity of low temperature operation has rendered such systems impractical for many applications. Our recently developed high intensity pump sources overcome this difficulty by effectively pumping to much higher inversion levels, allowing efficient operation at or near room temperature. Because the end-pumping technology is scalable in absolute power, the number of rare-earth ions and transitions that can be effectively accessed for use in practical DPSSL systems has grown tremendously. Unique laser systems for applications in fields such as medicine and remote sensing can now be simply realized.
\end{abstract}

We have also been involved in programs to evaluate the use of direct diodes for material processing applications. Here, diode radiation from an extended two-dimensional microlens-conditioned array is focused and delivered directly onto a work piece. Systems based on this concept can be utilized in the heat treating and hardening of metals.

Another application of microlens-conditioned laser diode arrays is in the direct coupling of their radiation to optical fibers. Direct diode-to-fiber coupling has recently been demonstrated for a medical application in which $22 \mathrm{~W}$ of CW 690 $\mathrm{nm}$ radiation was delivered from a microlens-conditioned stack of AlGaInP laser diode bars through a $1 \mathrm{~mm}$ core fused silica fiber. This approach used a simple and inexpensive $1 \mathrm{~cm}$ focal length lens to direct the microlens-conditioned radiation from the diode stack into the optical fiber.

Keywords: microlenses, laser diodes, diode pumped solid state lasers, diode-to-fiber coupling 


\section{FABRICATION OF MICROCHANNEL COOLED DIODE PACKAGES WITH INTEGRATED MICROLENSES}

LLNL has pioneered a low cost, high-average power modular laser diode array packaging technology that is compatible with the requirements of radiance conditioning using micro-cylindrical lenses. This technology enables the construction of large two-dimensional laser diode arrays using a simple stacking procedure. Figure 1 is a photograph of the present version of the LLNL laser diode package. Because it has been previously described $1,2,3$ only a brief overview will be given here. The approximate package dimensions are $2 \mathrm{~cm} \times 2 \mathrm{~cm} \times 0.75 \mathrm{~mm}$ thick. The package can hold up to 1.8 linear $\mathrm{cm}$ of bar material (visible underneath the wire bonds along the top edge of the photo). The circular holes on the right hand side of the package are internally connected and serve as the water inlet. Similarly, the holes on the left of the package are internally connected and serve as the water outlet. The central hexagonal hole allows a bolt to align modules stacked into two-dimensional arrays. The metallization pad visible in the photograph, to which the wire bonds are attached, serves as the electrical $n$-side diode contact. The diode bar is mounted p-side down on a metal pad that runs along the top edge of the package and then wraps around and connects to the bottom of the package (not visible in the photograph) which serves as the p-side contact.

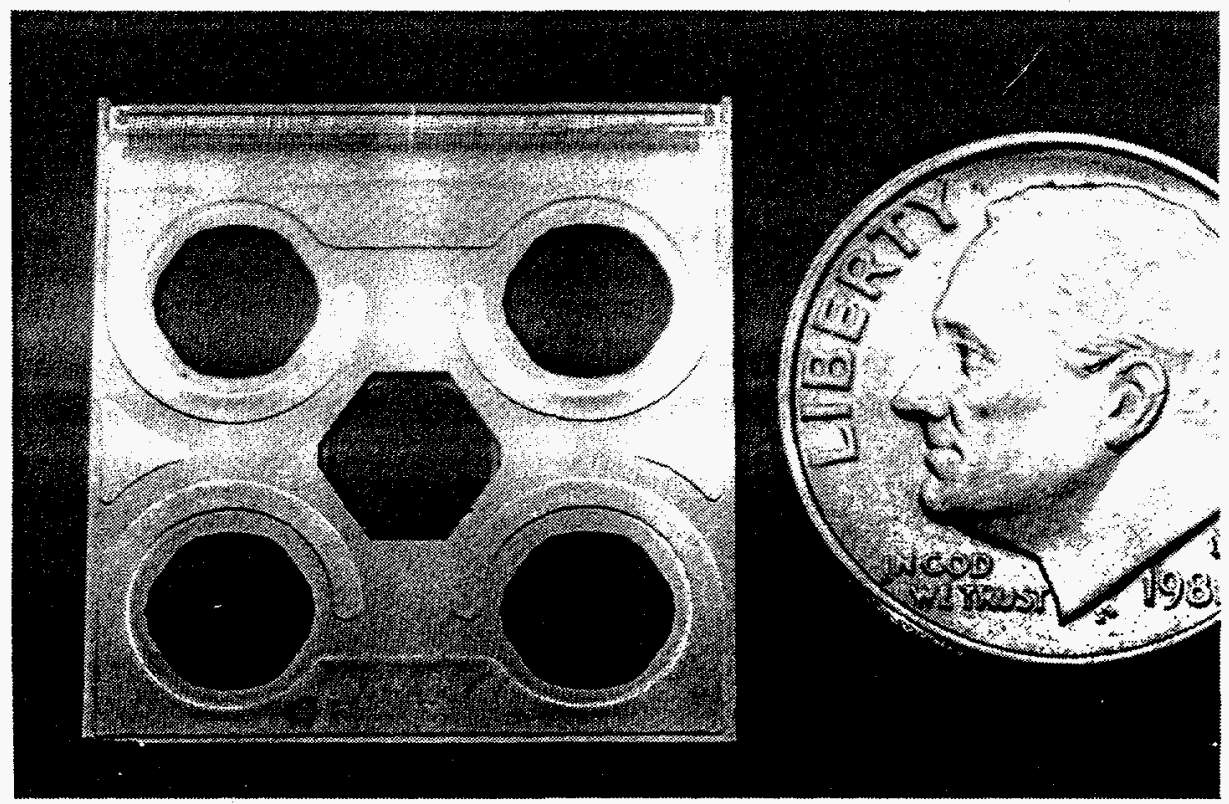

Figure 1. Photograph of the LLNL microchannel cooled laser diode package.

Figure 2a shows the laminated structure of the package in an exploded view. Two layers of silicon and one layer of borosilicate glass are used in the fabrication of the package. The borosilicate glass insert is sandwiched between the two pieces of etched silicon which comprise the manifold layer and the microchannel layer. All features on the glass piece are through-holes. On the silicon pieces, the central and four outlying holes are through-holes while the remaining patterned features are etched to a depth of between 150 and 200 microns. The sides of the silicon pieces (not shown) are featureless except for the through-holes and the canted front edge of the package. Figure $2 b$ is a cross-sectional view of the three layers showing the water flow path and the canted front package edge. The silicon layers and the central glass layer are approximately 250 microns thick. The etched features in the silicon bottom layer convert the flow of inlet cooling water entering the package through the two holes on the right hand side of Figure $2 \mathrm{a}$ into sheet flow toward the front of the package. Once at the front of the package, the water is turned toward the top layer of silicon through a slot that is ultrasonically milled into the central glass layer. After exiting the slot, the water enters the microchanneled region directly below the location of the diode bar. After exiting the microchannels, the water enters a sheet flow region in the top layer of silicon where it is directed back to the outlet holes on the left side of Figure 2a. The scanning electron micrograph of a cutaway package in Figure 3 shows these microchannels. In our present package design the channels are approximately 150 microns deep, 25 microns wide and $2 \mathrm{~mm}$ long. The purpose of the canted front edge of the package (visible in Figure $2 \mathrm{~b}$ ), which falls away from the horizontal at an angle of $35.3^{\circ}$, is to allow the emitted laser light to clear the package without 
having to reflect off any surface. As will be discussed later, this canted feature is also crucial for mounting cylindrical microlenses to focus the laser emission.
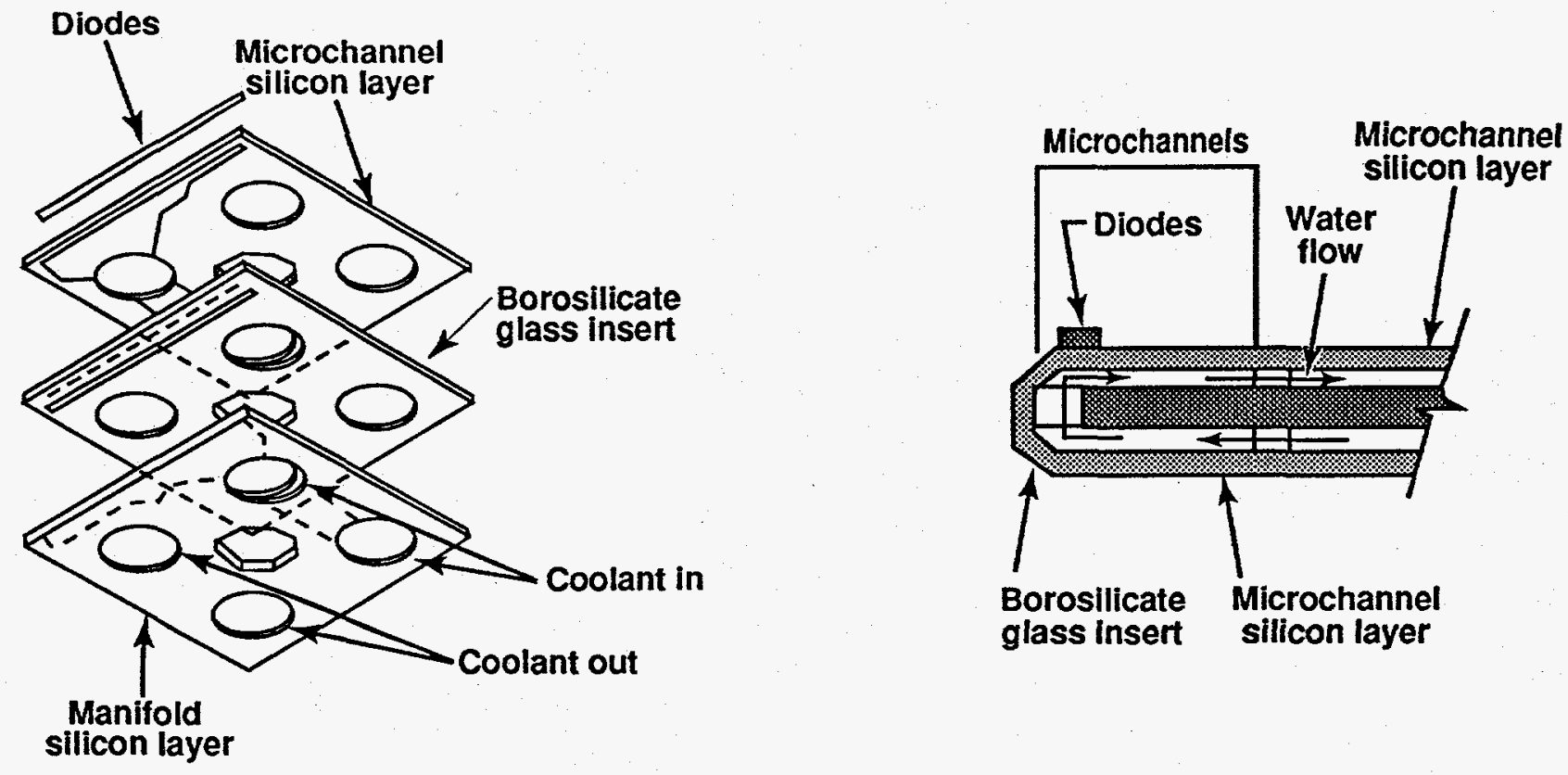

Figure 2. (a) Exploded view of the LLNL microchannel cooled package showing the three layers used in its construction. (b) Cross-sectional view of package.

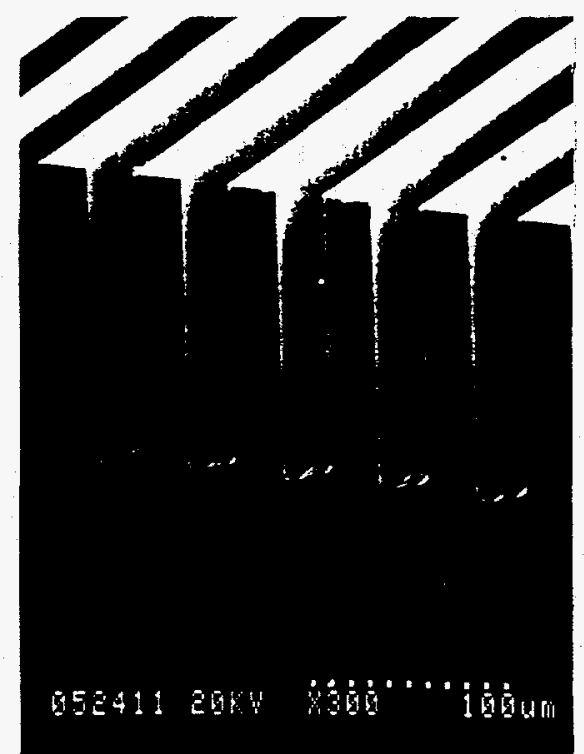

Figure 3. Scanning electron micrograph of the microchannel region in the packages top silicon layer.

The canted edge feature is formed by wet-etching, so its fabrication requires a particular orientation of the silicon wafer. To accommodate this, the microchannels are rotated $35.3^{\circ}$ from their orientation in our previous package design, 1 where they ran perpendicular to the front edge of the package. To prevent the larger and faster etching manifold features from etching through the silicon wafer before the slower-etching microchannels, an etch control technology has been developed. The depth of the feed plenums is 200 microns, which is $\sim 50$ microns deeper than the microchannels. 
The microchannel features in Figure 3 are responsible for the aggressive cooling capability of the LLNL package. In flowing liquid coolant loops, such as the one used in this package, it is generally true that the highest temperature rises are incurred in the stagnant fluid boundary layer that is always present between a flowing liquid and a solid. The source of these stagnant boundary layers is the no-slip boundary condition that exists at such interfaces. It is easy to see that these stagnant boundary layers become the thermal bottleneck of the thermal circuit when one realizes that the thermal conductivity of typical solids used in heatsinks is many times larger than the thermal conductivity of the typical fluids that flow through them. For example, the thermal conductivity of the silicon used in the LLNL package is $1.5 \mathrm{~W} / \mathrm{cm}-{ }^{\circ} \mathrm{C}$, which is 250 times larger than that of water at $0.0061 \mathrm{~W} / \mathrm{cm}-{ }^{\circ} \mathrm{C}$. To minimize the thermal boundary layer temperature rise, two competing techniques have been developed. One of these techniques makes use of high Reynolds number turbulent flow, such as used in the compact high intensity cooler $(\mathrm{CHIC})^{4}$, while the other makes use of laminar flow through microchannels 5 and is the approach we have adopted. The simplest way to view how microchannels drive down the boundary layer thickness is to note that as long as fluid is being forced through the channels, the boundary layer cannot be any thicker than half the channel width. This explains the use of the narrow channel; the boundary layer width scales with the physical channel width. One advantage of the microchannel approach over the CHIC approach is that the microchannels are very efficient in terms of their consumed hydraulic power because they use low Reynolds number (laminar) flow. This is in contrast to the turbulent flow found in CHIC coolers which generally requires higher flow velocities and larger pressure drops to achieve heat transfer coefficients comparable to those realized in the microchannel approach. The interested reader is referred to the excellent treatments in the literature that discuss the heat transfer physics and design optimization procedures for microchannel based cooling circuits. 6,7

Because silicon processing techniques have already been well developed, the package can be fabricated at low cost and can be easily implemented into a system. Figure 4 shows a drawing of the pattemed silicon layers used for the top microchannel and bottom manifold layer of the package and the glass insert used as the central layer. Presently we are using a 3" silicon wafer technology that allows six individual patterns to be etched on each wafer piece as shown. The approximate thicknesses of the silicon wafers and the central glass insert are 250 microns. The silicon wafers are (110) oriented; this allows the through holes, manifold features, microchannels, and canted front edge feature to be anisotropically etched in a single step. In this etching process an $800 \AA \mathrm{SiN}_{\mathrm{X}}$ layer is deposited on both wafer surfaces and patterned using a photolithographic process to define those areas that are to be etched. The wafers are etched in a $44 \%$ $\mathrm{KOH}$ solution for a predetermined time. The approximate etch rate is 4 microns/hour with the exact rate being dependent on the feature size being etched. The central glass insert is fabricated using an ultrasonic machining technique. The three wafer layers shown in Figure 4 are joined using an anodic bonding procedure. ${ }^{8}$ In this process the three layers are placed in contact with each other and heated in an oven to $560^{\circ} \mathrm{C}$, the annealing temperature of the borosilicate glass insert. Using the glass as a negative electrode and the two silicon layers as positive electrodes, an increasing voltage difference is applied up to $500 \mathrm{~V}$ over a period of 45 minutes. The electrostatic force pulls the glass and silicon into intimate contact, forming a very strong bond at the interface. After the bonding is completed, the parts are annealed at high temperature for approximately two hours before being removed from the oven. After cooling, the wafer assemblies are cut into individual packages using a dicing saw.

Standard thin film metallizations are applied to the coolers using a shadow mask. Starting at the silicon and going outward, the metallization layers are $1000 \AA$ of $\mathrm{Ti}, 20 \mu \mathrm{m}$ of $\mathrm{Au}, 1000 \AA$ of $\mathrm{Pt}$, and $1000 \AA$ of $\mathrm{Au}$. The thick Au layer ensures a low resistance current path for the up to $250 \mathrm{amps}$ of current that run through the packages. As already discussed, the package is metallized on its top, bottom, and front edge, with a break in the metallization that runs just below the diode bar. This break is located just underneath the wire bonds (in Figure 1) to provide isolation for the n-side contact where the wire bonds are attached. The diode bar is soldered to the package using a $3 \mu \mathrm{m}$ thick layer of evaporated In. The final step in the package production is the wire bonding from the $n$-side of the bar to the packages $n$-side contact using approximately two hundred, 1.25-mil-diameter Au wires.

Because diode radiation is generated in a waveguide structure whose height is only slightly larger than the radiation's wavelength, the fast axis divergence angle of the radiation (perpendicular to the p-n junction plane) can be as large as $60^{\circ}$ (FWHM). For many applications, this is problematic because large two-dimensional arrays do not allow efficient light concentration in the fast axis direction without some type of optical conditioning to reduce the fast axis divergence. To address this problem LLNL has developed an inexpensive cylindrical microlens technology that allows the efficient collection and conditioning of the diode radiation in the fast axis direction. ${ }^{9}$ Figure 5 shows a cross-sectional view of an LLNL laser diode package with a conditioning microlens attached. Our latest generation microlens is shaped to rest on the canted front edge of the package in front of the laser diode bar with its flat surface toward the emitting diode. The lens collects the light emitted from the diode with up to a $60^{\circ}$ (1 Rad) divergence angle and collimates it to approximately $0.6^{\circ}$ (10 mRad). The LLNL microchannel cooled packages can be stacked at a pitch of 10 per $\mathrm{cm}$ to form two-dimensional arrays capable of generating average power pump intensities of $1 \mathrm{~kW} / \mathrm{cm}^{2}$ at the array aperture. The great utility of the 
microlens technology is that when large two-dimensional arrays are built by stacking packages, the effective radiance in the fast axis direction is increased by a factor of 100 . This allows large stacks to be focused down to spots in this fast axis dimension that are a factor of 100 smaller in spatial extent than possible without optical conditioning.

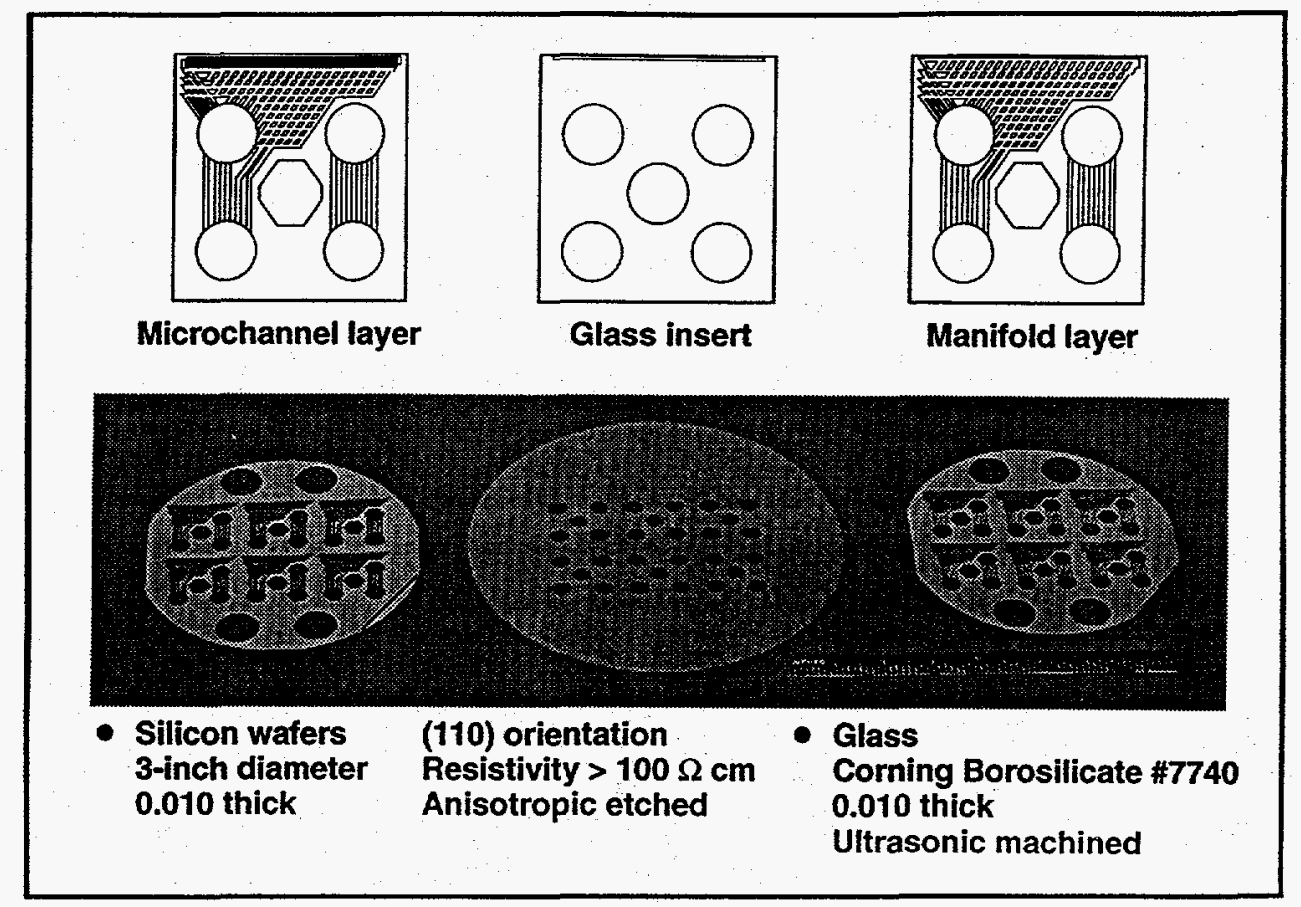

Figure 4. The three layers of a microchannel cooler package are shown along the top row. The features in the silicon microchannel and manifold layers are fabricated using an anisotropic etching technique while the features in the glass layer are fabricated using an ultrasonic milling technique. The bottom row shows the two silicon wafers and the single glass wafer that are used in the manufacture of the cooler assemblies.

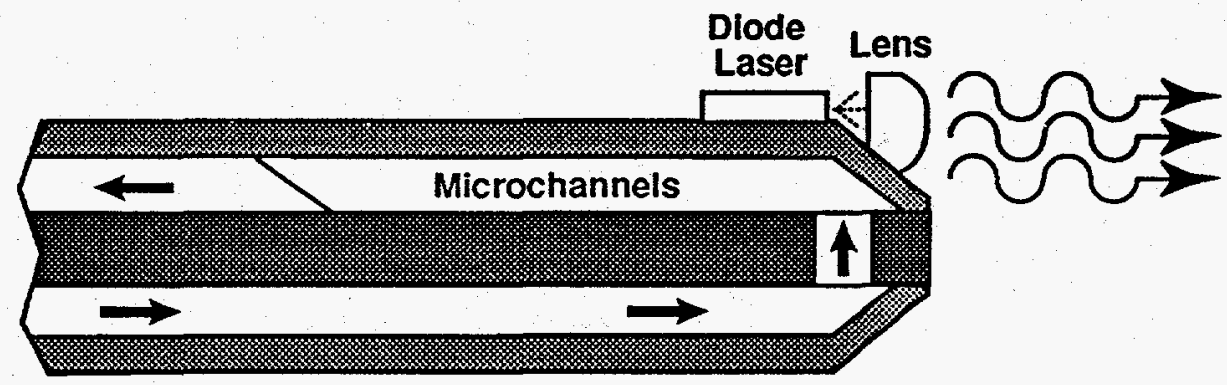

Figure 5. Cross-sectional view of present LLNL laser diode package with a conditioning microlens attached.

Key to the radiance conditioning technology described here is the ability to inexpensively fabricate very fast cylindrical microlenses that efficiently collect the emitted diode radiation and then collimate it. At LLNL, a technique has been demonstrated in which high quality microlenses can be drawn from a shaped optical fiber preform. In cross-sectional shape, the fiber preform is ground so that it has a profile identical to that desired on the microlens although at a scale that is approximately 75 time larger in its transverse dimensions. In the pulling of the optical fiber from the preform, the temperature is kept low enough so that the resulting fiber retains the desired cross-sectional shape of the preform. Pulling the shaped preforms into shaped cylindrical microlenses is done by Collimated Holes, Inc. located in Campbell, California. The final shaped-fiber diameter is approximately 350 microns, yielding approximately $1 \mathrm{~km}$ of fiber lens from the preform. The heating and drawing operation reduce the cross sectional dimensions of the original preform by $\sim 75$ times, thereby reducing any surface imperfections in the preform by that amount. Referenced to the wavelength of light, it is clear that shrinking the surface errors during the fabrication process facilitates the production of lenses having high optical quality. 10 Such fiber micro-cylindrical lenses are now commercially available from Blue Sky Research located in San Jose, California. After the lens is drawn, it is cut into short $30 \mathrm{~cm}$ lengths and AR coatings are applied to both the input and 
output faces. These coated lengths are then cut into $2 \mathrm{~cm}$ long pieces and individually attached to the LLNL diode packages. This lens attachment is performed actively while the package is emitting light. To properly locate a lens, a camera is used to view the far field radiation pattern from the package on a screen with a $1 \mathrm{~cm}$ grid located $1 \mathrm{~m}$ from the laser diode bar. With the lens held in a vacuum chuck it is moved using an $(x, y, z)$ electronically controlled stepper motor positioning apparatus until the desired far field emission pattern is seen. Figure 6 shows an example of such a far field radiation pattern from a package with a properly located and attached lens. The $1 \mathrm{~cm}$ high dimension of the illuminated region ( 1 box) is a measure of the $10 \mathrm{mRad}$ beam divergence of the microlens-conditioned diode fast-axis radiation. At this point, hypodermic needles are used to carefully deposit a low-shrinkage UV-cured glue on each end of the lens. Finally, a UV lamp is turned on for several seconds to cure the glue. A low shrinkage glue is necessary to prevent the lens from shifting from its optimally located position during the curing process. A skilled operator can attach a lens in under two minutes, which only marginally impacts the production cost of such packages.

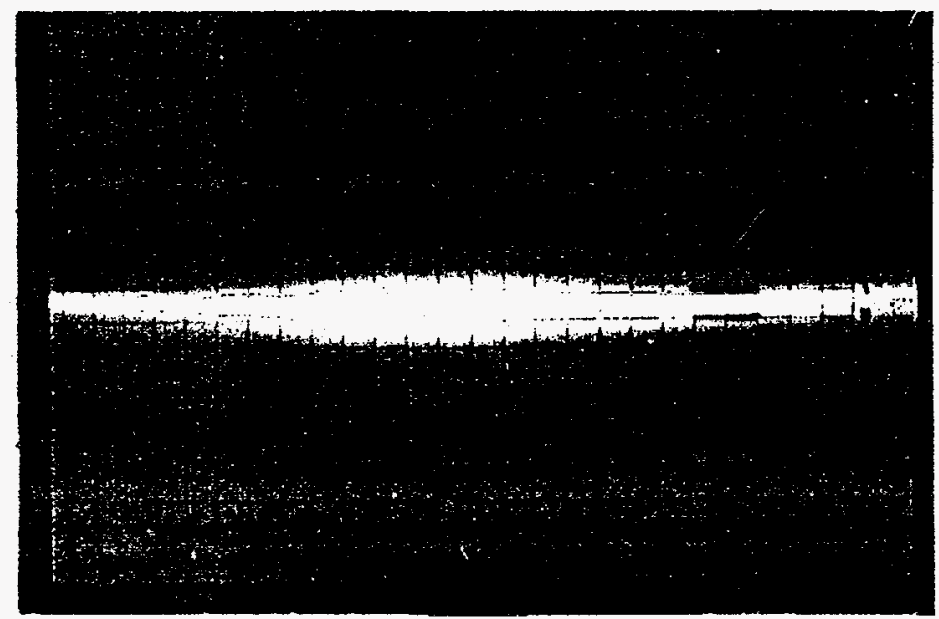

Figure 6. Photograph of the farfield emission pattern from a microlens-conditioned laser diode array. The squares on the screen are $1 \mathrm{~cm}$ on a side and the screen was located $1 \mathrm{~m}$ from the emitting array.

Critical to obtaining good optical performance from the microlens-conditioned packages is the flatness of the laser diode bar (absence of so called "smile") when it is attached to the top silicon surface of the package. Along the length of the diode bar (up to $1.8 \mathrm{~cm}$ ) the flatness should be within \pm 2 micron. This flatness requirement is strict because of the short effective focal length of the microlenses. Our present generation lens shown in profile of Figure 5 has an effective focal length of approximately 250 microns. This means that if the string of emitting apertures along the diode bar deviate from a straight line by only $2.5 \mu \mathrm{m}$, due to non-planarity of the submount, then the resulting collimated diode radiation will be angularly deviated by $10 \mathrm{mRad}$, the same as the far field beam divergence of the conditioned diode radiation. Since the silicon wafers used in the LLNL package production are polished, this flatness requirement is easily met, illustrating another advantage of this silicon processing technology. The same flatness specifications are much harder to meet when using either $\mathrm{BeO}$ or copper because of difficulties in machining these materials to the required tolerances.

Figure 7a shows a LLNL package along with a matching $250 \mu \mathrm{m}$ thick conductive silicone elastomer gasket. Such package-gasket assemblies are stacked one on top of another using the central hole to locate on a bolt as shown in Figure 7b. The overall thickness of such package-gasket assemblies is $1 \mathrm{~mm}$, giving a stacking pitch of 10 packages per $\mathrm{cm}$. Using this stacking procedure arbitrarily-large two-dimensional apertures can be constructed. The central locating bolt is used to hold the stack in compression when it is completed. In such stacks the conductive gaskets provide electrical continuity from package to package as well as hydraulic sealing between the packages. Regardless of the length of the stack, water connections from package to package are made automatically by the gaskets so that a finished stack simply has one water inlet and one water outlet. This feature greatly simplifies the integration of such large two-dimensional diode pump arrays into DPSSL systems. One of the strengths of the LLNL packaging approach is the flexibility it brings to the construction of two-dimensional laser diode arrays. A single base package allows the construction of arbitrarily large, close packed, high average power laser diode arrays with microlens-conditioning. Because such a broad spectrum of laser diode applications can be addressed using this single modular package approach with the option of integrated microlenses, there has recently developed commercial interest in the technology. 

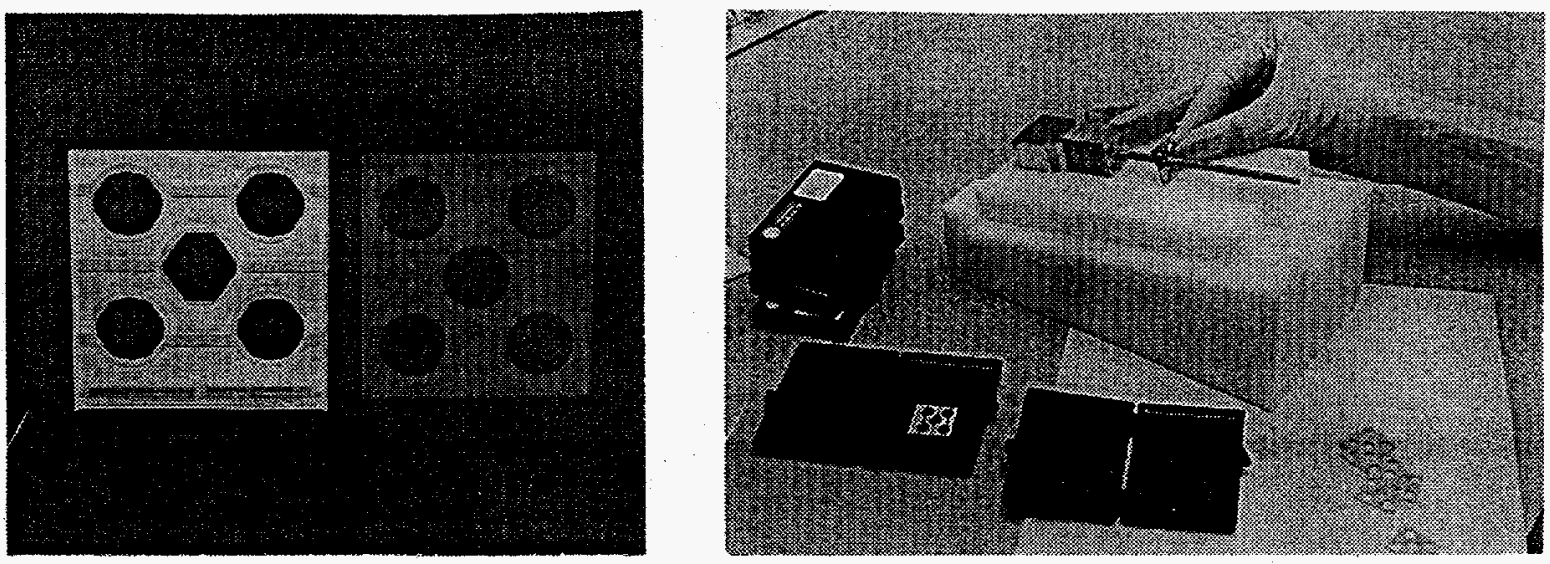

Figure 7. (a) Photograph of the LLNL package with its matching conductive silicone elastomer gasket. (b) A single bolt is used to locate individual packages with alternating conductive silicone elastomer gaskets in the construction of large two-dimensional diode arrays.

\section{MICROLENS DESIGN AND FABRICATION}

Figure 8 shows a microchannel cooled module with one of the original LLNL designed microlenses attached. ${ }^{10}$ In the geometry shown, the diverging light is collimated by the shaped entrance face leading to a very simple hyperbolic lens design, i.e., the entrance face profile is a hyperbola. Unfortunately, angularly-extreme incident rays suffer from large Fresnel reflective losses because of the large angles they make with the incident curved surface. If the lens geometry is conceptually reversed so that the diverging light first enters a planar surface and exits through a curved surface, this entrance face loss problem is mitigated but now the curved exit face is somewhat more complicated to calculate as it is no longer a simple conic. Because we wanted to push the NA of our cylindrical microlenses to values approaching 1 and maximize their collection efficiency we have designed our latest generation of lenses with a flat input face as shown previously in Figure 5.

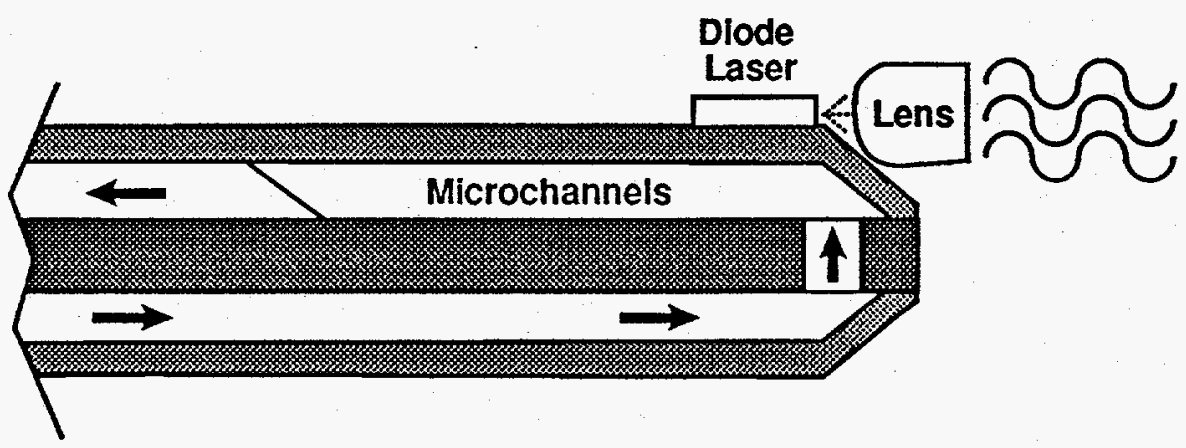

Figure 8. Cross-sectional view of LLNL laser diode package with an original design conditioning microlens attached.

Figure 9 shows the coordinate system used in calculating the present microlens profile. The diode aperture is located at the origin of the coordinate system and rays of light leaving the origin are parameterized by the angle $\theta$ which they make with the $x$-axis. The flat input face of the lens is located at $x=a$, while the curved output face intersects the $x$-axis at $x=b$. Working in the first quadrant, the curved profile of the microlens denoted by the function $y=f(x)$ can be determined using a variational calculation based on Fermat's principle in which a ray is tracked from the origin through the lens to a focus point at infinity. As shown in the following derivation, the desired surface of the optic is complicated but still easily calculated numerically. To this end a coupled system of linear differential equations is derived in which the lens profile is described parametrically in terms of $\theta$ by the coordinate pair $(x(\theta), y(\theta))$. 


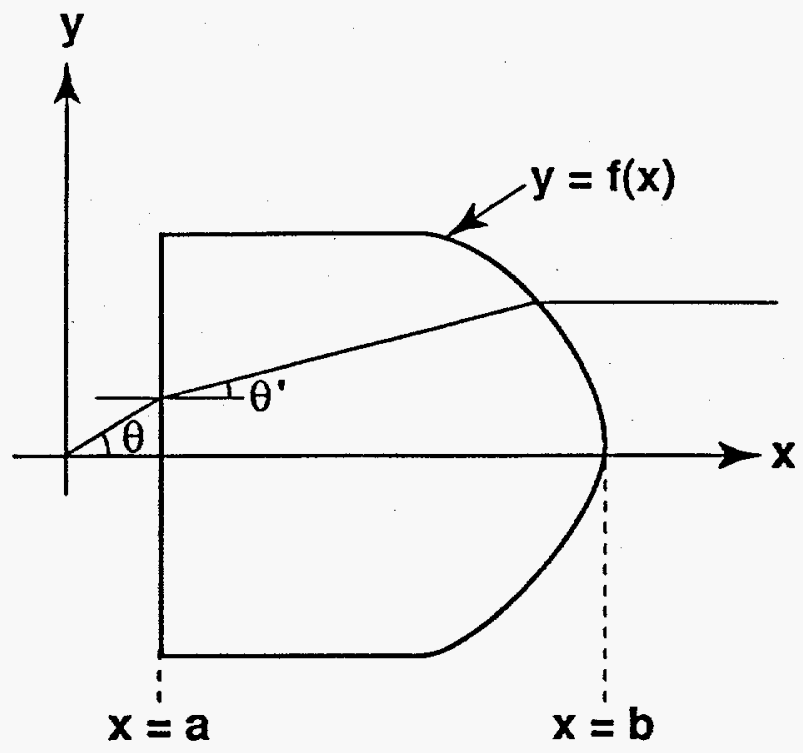

Figure 9. Coordinate system used in calculating the profile of cylindrical microlenses. The emitting diode aperture is located at the origin in this figure.

A ray launched from the origin intersects the flat input face of the lens at the point $\left(x_{1}, y_{1}\right)$ given by,

$$
\begin{aligned}
& x_{1}=a \\
& y_{1}=a \cdot \tan (\theta)
\end{aligned}
$$

Calling $n$ the index of refraction of the lens material, the angle $\theta^{\prime}$ in Figure 13 is determined from Snell's law,

$$
\sin (\theta)=n \cdot \sin (\theta)
$$

If the intersection of this ray with the curved surface of the lens is now denoted by $(x, y)$, the constraint placed on $(x, y)$ by the slope of the ray inside the lens is,

$$
\frac{y-a \cdot \tan (\theta)}{x-a}=\tan (\theta)
$$

Using (0.2) to eliminate $\theta^{\prime}$ from (0.3) gives,

$$
\frac{y-a \cdot \tan (\theta)}{x-a}=\frac{\sin (\theta)}{\sqrt{n^{2}-\sin ^{2}(\theta)}}
$$

Calling $d$ the optical path length of this ray from the origin to infinity, $d$ is given by,

$$
d=a \sqrt{1+\tan ^{2}(\theta)}+m \sqrt{(x-a)^{2}+(y-a \cdot \tan (\theta))^{2}}+\sqrt{\left(x_{\infty}-x\right)^{2}+(y-a \cdot \tan (\theta))^{2}}
$$

Approximating the last radical in $(0.5)$ by $x_{\infty}-x$ gives,

$$
d=a \sqrt{1+\tan ^{2}(\theta)}+n \sqrt{(x-a)^{2}+(y-a \cdot \tan (\theta))^{2}}+x_{\infty}-x .
$$

Equations (0.4) and (0.6) represent two fundamental design equations for the lens surface $y=f(x)$. Fermat's principle requires that all rays starting at the origin and ending at a focus at infinity have equal optical path lengths. Treating $x$ and $y$ 
in Equations (0.4) and (0.6) as funclions of the parametric variable $\theta$ and implicitly differentiating with respect to $\theta$ leads to a coupled set of linear equations in $\mathrm{d} x / \mathrm{d} \theta$ and $\mathrm{d} y / \mathrm{d} \theta$,

$$
\begin{aligned}
& {\left[\begin{array}{cc}
(x-a)-\frac{1}{n} \sqrt{(x-a)^{2}+(y-a \cdot \tan (\theta))^{2}} & y-a \cdot \tan (\theta) \\
\frac{-(y-a \cdot \tan (\theta))}{(x-a)^{2}} & \frac{1}{x-a}
\end{array}\right]\left[\begin{array}{l}
\frac{d x}{d \theta} \\
\frac{d y}{d \theta}
\end{array}\right]=} \\
& {\left[\begin{array}{c}
-\frac{1}{n} \frac{a \cdot \sin (\theta)}{\cos ^{2}(\theta)} \sqrt{(x-a)^{2}+(y-a \cdot \tan (\theta))^{2}}+(y-a \cdot \tan (\theta)) \frac{a}{\cos ^{2}(\theta)} \\
\frac{a}{(x-a) \cos ^{2}(\theta)}+\frac{n^{2} \cos (\theta)}{\left(n^{2}-\sin ^{2}(\theta)\right)^{3 / 2}}
\end{array}\right]}
\end{aligned}
$$

In deriving (0.7), Fermat's principle has been invoked by setting $d d / d \theta=0$ when performing the differentiation in (0.6). Using the initial condition that at $\theta=0, x=b$ and $y=0$ it is a simple numerical calculation to generate the form of the curved lens surface. In this calculation the working distance $a$ of the lens (see Figure 9) is treated as a constant. Large numerical aperture diffraction limited lens profiles can be calculated by incrementally stepping through the angle $\theta$ and calculating the $x$ and $y$ values at each value of $\theta$ using $(0.7)$ and the $x$ and $y$ values at the previous $\theta$. Following this procedure we generate a table of $(r, \theta)$ values describing the curved surface which is then used as the control input to a computer controlled milling machine in the fabrication of the shaped fiber-preform.

\section{APPLICATIONS OF MICROLENS-CONDITIONED LASER DIODE ARRAYS}

The average power performance capability of semiconductor laser diode arrays has improved dramatically over the past several years. 11 These performance improvements combined with cost reductions pursued by LLNL and others in the fabrication and packaging of diode laser arrays have continued to reduce the price per average watt of diode radiation. Presently, we are at the point where the manufacturers of commercial high average power solid state lasers can now seriously consider the replacement of their flashlamp pumps with laser diode pump sources.

Chief among the many advantages of using laser diode arrays as replacements for flashlamps in the excitation of solid state lasers is the ability of laser diode arrays to be focused to high intensities for use in the end-pumping of laser crystals. This capability has significantly expanded the number of ions and transitions that have been demonstrated to be useful as lasants. As the number of lasant ions and accessible transitions has grown, so has the diversity in the wavelength of DPSSL systems and the number of applications for which they are being pursued.

At present, DPSSLs are being contemplated and developed for atmospheric and space based LIDAR systems, underwater illumination and sensing, wind shear sensing, remote sensing of chemical species, lithographic processing, medical applications, and electronic and material processing applications. The ability to focus or condense the output from semiconductor diode laser arrays to high intensities is important for the efficient operation of lasers in many of these applications because of the high pump intensities required to efficiently excite the laser ions to useful levels.

Until now, one draw back the diode end-pumped architecture has suffered from has been the limited number of diode apertures that could be combined into a single focused beam. This has limited the peak and average power scaling capability of the end-pumping architecture and restricted the number of systems in which it could be usefully implemented. In the past, when peak or average power scaling was required in a DPSSL it was necessary to use a transverse pump geometry. A transverse pump geometry is one in which the diode arrays are placed adjacent to the laser crystal, thereby essentially flooding the crystal with pump radiation. In this approach, no attempt is made to focus the output radiation of the diodes into a single spot and the pump intensity at the crystal is limited to approximately the pump intensity at the diode array face (which today is at most several $\mathrm{kW} / \mathrm{cm}^{2}$ ). These low pump intensities have restricted the use of the transverse pump geometry to the $\mathrm{Nd}$ ion.

An end-pump geometry which can generate pump intensities up to $100 \mathrm{~kW} / \mathrm{cm}^{2}$ and be simply and easily scaled in peak and average power into the $\mathrm{kW}$ range has recently been advanced by LLNL. Previously, the stumbling block in the 
development of a scalable diode end-pumping architecture has been the intrinsically low effective radiance of the twodimensional emitting apertures constructed from a stack of laser diode array bars. The low effective radiance of diode arrays is due to the high $60^{\circ}$ divergence angle of their emitted radiation in the fast axis direction. As previously described the key to overcoming the low radiance in the fast axis direction is the optical conditioning of the diode radiation from individual bars using precision cylindrical microlens. In such stacks, the microlens conditioning decreases the fast axis divergence angle by approximately a factor of 100 . It is this decrease in divergence and the accompanying ability to focus the radiation to a smaller spot that enables the output radiation from large two-dimensional diode stacks to be efficiently delivered to the end of rod lasers.

Figure 10 shows a drawing of an end-pumped rod laser. The radiation from the microlens-conditioned stack of laser diode arrays is delivered to the laser rod by a device called a lensing duct. This optical component can be fabricated from any transparent optical material and relies on lensing at its curved input face and total internal reflection on its canted planar faces to channel the diode pump radiation to the laser diode bar. The best way to visualize the operation of the lensing duct is to view it as an immersion lens; i.e., its length $l$, input radius of curvature $R$, and index of refraction $n$ are related by $l=R n /(n-1)$. Based on measurements and modeling, the transfer efficiency of the lensing duct for the microlens-conditioned radiation from a $6 \mathrm{~cm} \mathrm{x} 1 \mathrm{~cm}$ diode array aperture ( $6 \mathrm{~kW}$ aperture) to a $3.2 \mathrm{~mm}$ diameter laser rod is in excess of $90 \%$. The advantage of the lens duct over a simple lens is greater efficiency. Because the diode aperture represents an extended incoherent source of radiation, imaging a large array via a simple lens generally overfills the input aperture of a small rod many times. With the lensing duct the radiation that would be delivered outside the aperture of the rod can still be efficiently channeled to the rod by reflecting off its canted planar sides.

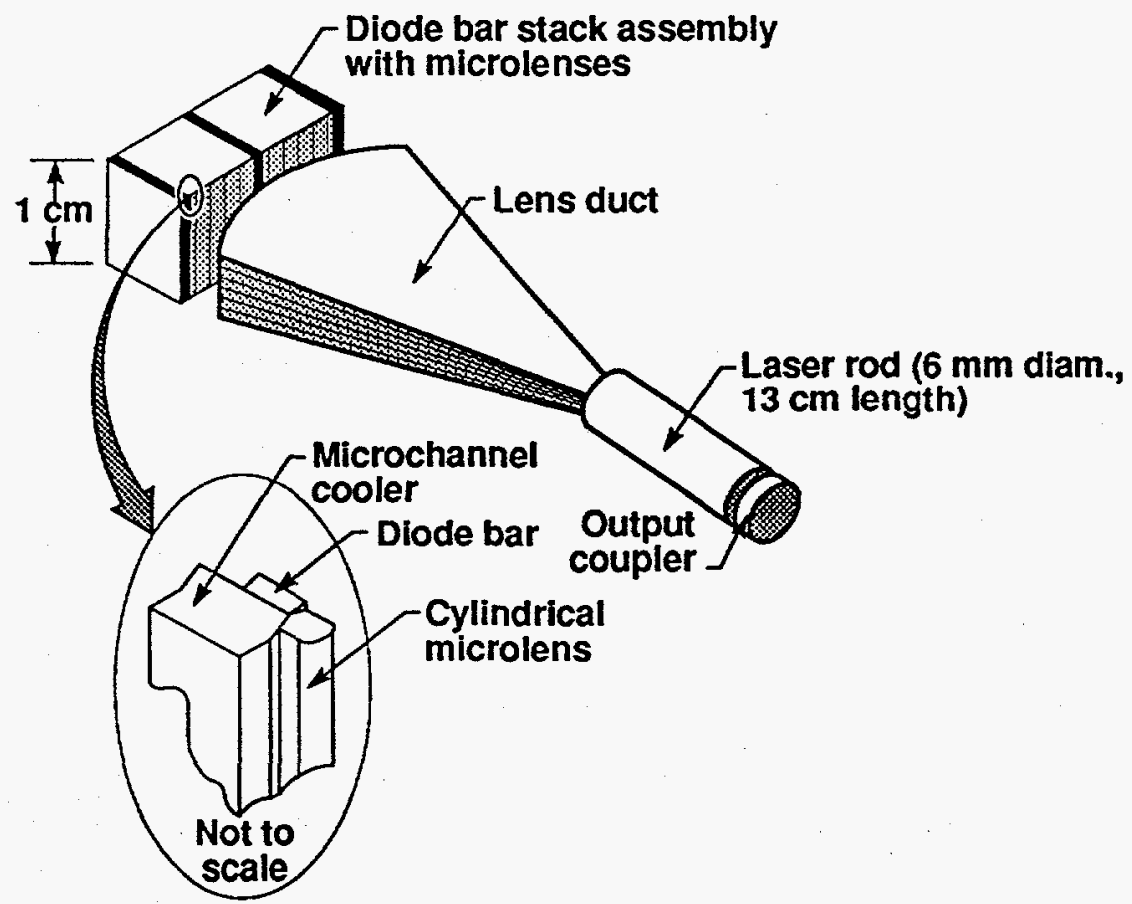

Figure 10. The lensing duct's total internal reflection efficiently channels the pump radiation to the laser bar.

The ability to build high density, high average power laser diode arrays, and the microlens conditioning/lensing duct delivery that enables the outputs of large two-dimensional arrays to be efficiently delivered to the end of rod lasers, defines the LLNL scalable end-pumping architecture. To date this technology has been used to build a $100 \mathrm{~mJ}$ Q-switched Nd:YLF laser oscillator 12 for use in atmospheric and space-based LIDAR systems, and a wing-pumped Cr:LiSAF laser using 770 $\mathrm{nm}$ AlGaAs laser diode arrays ${ }^{13}$. Presently, the end-pumping technology is being pursued in the demonstration of a $2 \mu \mathrm{m}$ Tm:YAG laser for medical applications.

Figure 11 depicts the energy levels of the $\mathrm{Tm}^{3+}$ ion that are utilized in the $2 \mu \mathrm{m}$ Tm:YAG laser. Population is promoted from the ground ${ }^{3} \mathrm{H}_{6}$ manifold to the ${ }^{3} \mathrm{~F}_{4}$ manifold by pumping at $785 \mathrm{~nm}$ with AlGaAs diodes. This population then relaxes to the ${ }^{3} \mathrm{H}_{4}$ manifold which contains the initial laser Stark level. The terminal laser Stark level is contained in 
the ground ${ }^{3} \mathrm{H}_{6}$ manifold. Spectroscopic laser schemes such as this in which the terminal laser level lies in the ground state manifold are commonly referred to as quasi-three-level lasers. Because the terminal laser level is thermally coupled to all the Stark levels in the ground state manifold and not significantly separated from the lowest lying Stark level, it contains some fraction of the ground state manifold population as determined by the Boltzmann distribution. This results in absorption by the sample at the laser's emission wavelength that must be overcome by pumping. Traditionally, the presence of such re-absorption losses in a laser have usually meant that the laser had to be operated at low temperatures such that the population present in the terminal laser Stark level at room temperature was frozen out. Such low temperature operation was the only practical way to lower the threshold of many of these three level systems enough to overcome the deleterious effects of ground state absorption and enable lasing with conventional pump excitation sources such as flash lamps. With the advent of semiconductor laser diode arrays and their ability to generate very high pump intensities, many of the systems that were previously only possible at low temperatures are now not only possible, but practical, at room temperature. This room temperature operation results from the ability of laser diode arrays to pump laser samples to much higher excited state fractions than was previously possible, thus overcoming the ground state absorption losses by brute force.

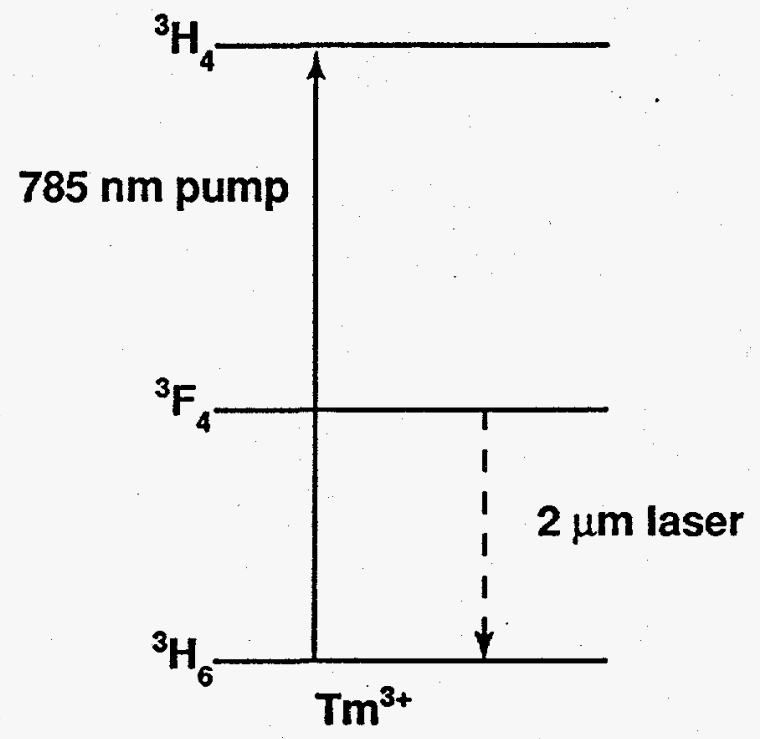

Figure 11. Energy levels of $\mathrm{Tm}^{3+}$ used in our 2 micron laser.

Figure 12 shows several photographs of the Tm:YAG laser that is presently under development as well as a drawing indicating the various parts of the laser (visible in the photographs). In particular, the microchannel-cooled, microlensconditioned stack of laser diode bars and the lensing duct which define the scalable diode end-pumping technology are shown. The stack of laser diode arrays consists of 25 packages with each package containing 1 linear $\mathrm{cm}$ of diode bar material. Cylindrical microlenses collimate the output of each diode laser bar, allowing it to be efficiently delivered to the end of the $5 \mathrm{~cm}$ long by $3.2 \mathrm{~mm}$ diameter lightly-doped Tm:YAG rod. The lensing duct serves to channel the radiation from the diode arrays to the rod using a combination of lensing at its curved input face and total internal reflection (TIR) on its canted planar sides. The barrel of the YAG rod is polished so that the delivered pump light, confined within the rod by TIR, can be channeled down the rod as it is absorbed by the Tm.

In order to assess thermal management issues in the YAG rod, particularly at its pump input end, a breadboard has been constructed an operated using an $808 \mathrm{~nm}$ pumped Nd:YAG rod lasing at $1.06 \mu \mathrm{m}$. This breadboard has been operated reliably at $25 \mathrm{~W}$ of continuous output power using a Nd:YAG rod that was $2 \mathrm{~cm}$ long by $3.2 \mathrm{~mm}$ in diameter. Of particular importance to the reliable operation of lasers using this end-pumping architecture is ensuring that the TIR of the pump light within the laser rod is not frustrated. This requires that careful attention be paid to the o-rings which are used to seal the rod in its water-cooled jacket. Conventional black o-rings against the polished barrel of the YAG rod can effectively frustrate TIR of the pump light and lead to local heating of the rod. At present, the Tm:YAG laser generates $5.5 \mathrm{~W}$ of CW $2 \mu \mathrm{m}$ radiation. 

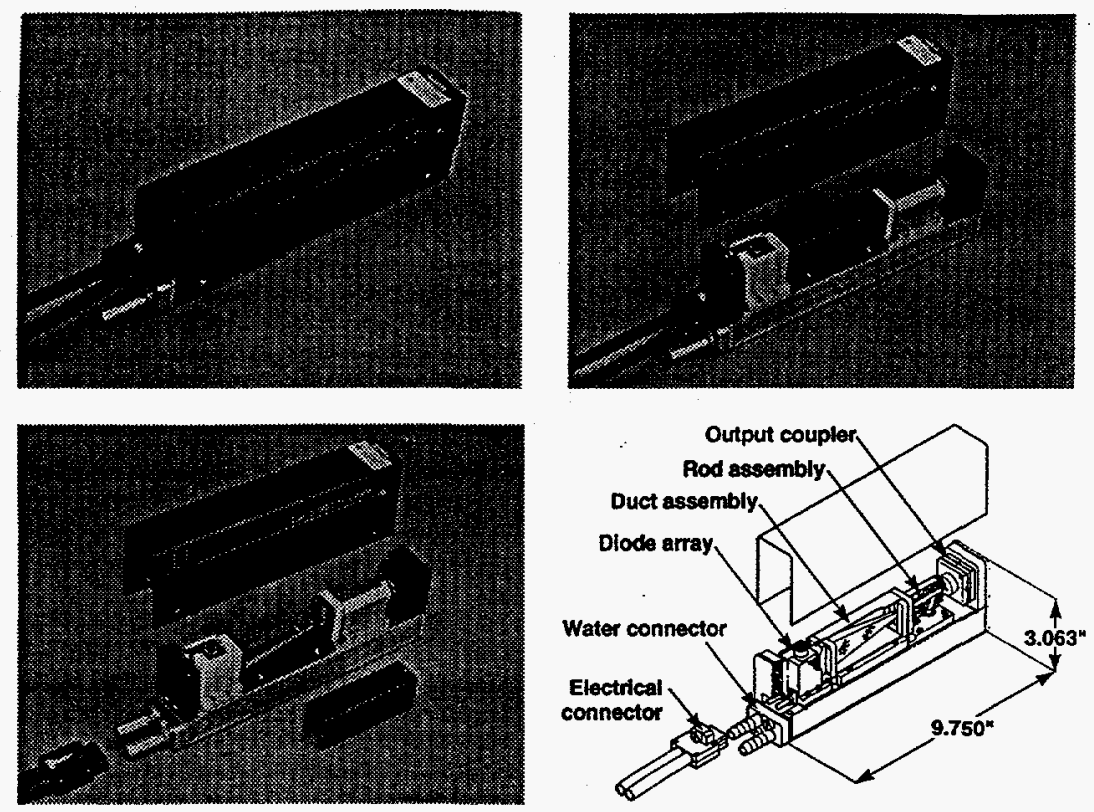

Figure 12. Photographs and drawing of LLNL Tm ${ }^{3+}$ :YAG laser.

One of the very promising commercial developments emerging from this technology is in the area of high average power DPSSL systems for material processing using Yb:YAG as the gain element. The high intensities that can be generated with the LLNL end-pumping technology offer an opportunity to overcome the ground state re-absorption problem of the $\mathrm{Yb}^{3+}$ ion. At the same time, advantage can be taken of the very small thermal power generation parameter of the $\mathrm{Yb}^{3}+$ ion by using the high average power capability of the LLNL diode laser technology to implement a high average power DPSSL system using a very simple rod laser approach. Because such a Yb-based DPSSL produces a fiber delivery compatible output at $1 \mu \mathrm{m}$, it is receiving much attention from the industrial laser material processing community.

Another application of radiance conditioned diode arrays is shown in Figure 13. Here a two-dimensional aperture formed from a stack of microlens-conditioned packages has its output radiation delivered into an optical fiber via a simple focusing lens. Figure 14 shows a photograph of such a diode-to-fiber coupling unit. In this hardware, a five package stack of $5 \mathrm{~mm}$ long AlGaInP laser diode bars emitting at $690 \mathrm{~nm}$ is delivered into a $0.37 \mathrm{NA}, 1 \mathrm{~mm}$ core fused silica optical fiber. A simple $1 \mathrm{~cm}$ focal length spherical lens is used to focus the stack output into the fiber. In this application the slow axis (unconditioned direction) divergence angle of the emitted laser diode radiation limits the usable diode bar length to $\sim 5 \mathrm{~mm}$. The measured coupling efficiency of the entire post-diode optics chain, including microlenses, $1 \mathrm{~cm}$ focal length spherical lens, and uncoated optical fiber, is $58 \%$ at the output end of the fiber. One of the advantages of the diode-to-fiber coupling scheme shown here as opposed to the more prevalent commercial approach in which individual "fiberlets" are butt-coupled to emitting apertures and then rebundled into a single larger fiber 14 is the flexibility and robustness of the package. The technology using the individual butt-coupled fiberlets requires a precision alignment of the fiberlets to the emitting apertures and generally leads to a sealed unit in which the. laser diode array and fiberlets cannot be easily separated if it becomes necessary to replace either the diode or the fiber. The LLNL approach allows the use of an SMA coupled fiber that can be quickly and easily replaced using its socketed connector. This is advantageous for medical applications where it may be necessary to replace the fiber on a routine basis. Additionally, in the LLNL approach the entire laser diode array can be removed and serviced easily and quickly because it does not require the precision alignment that characterizes the fiberlet approach to coupling. Thus, a single unit can provide multiple wavelengths at different times by simply swapping in and out different diode stacks.

At present, the device shown in Figure 14 has delivered $22 \mathrm{~W}$ of $\mathrm{CW} 690 \mathrm{~nm}$ radiation at the end of the fiber. This particular piece of hardware was built for a photodynamic therapy (PDT) application. Additional applications of such units are anticipated in the field of material processing where it is envisioned they could deliver $\sim 50 \mathrm{~W}$ of $\mathrm{CW}$ radiation using AlGaAs or InGaAs laser diode arrays. Because of the non-zero divergence of our microlens-conditioned laser diode source in both the fast axis and slow axis directions, the scalability of our approach is essentially limited to the stack array size described here ( $5 \mathrm{~mm} \times 5 \mathrm{~mm}$ aperture) for the $1 \mathrm{~mm}$ core, large numerical aperture fibers used. 


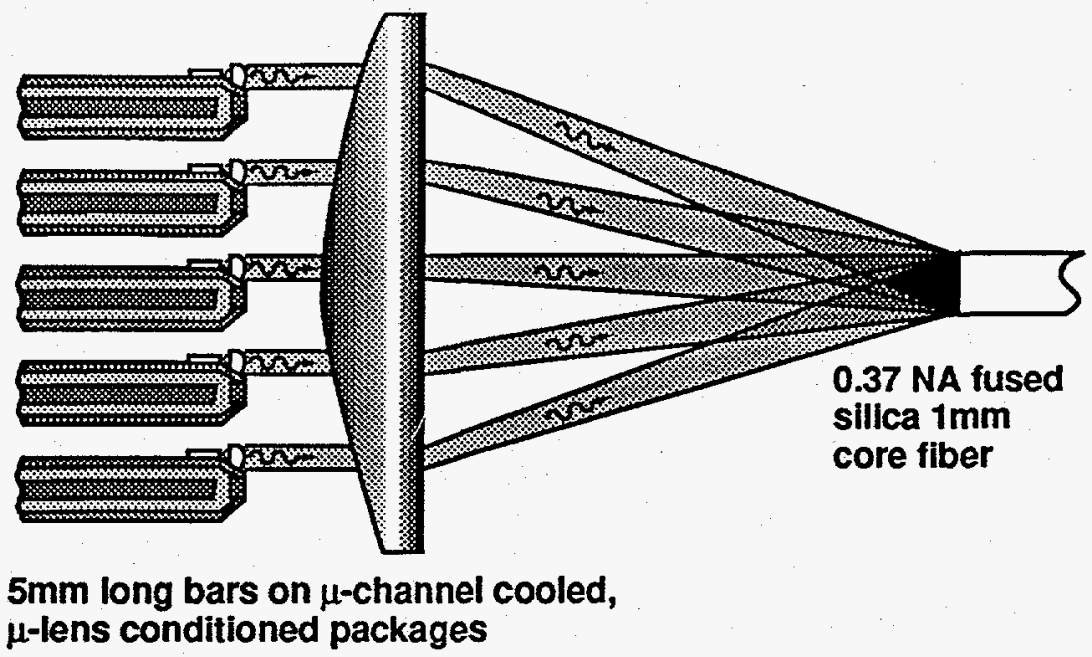

Figure 13. Schematic drawing illustrating the use of a simple lens to directly couple radiation from an extended microlens-conditioned stack of laser diode arrays into an optical fiber.

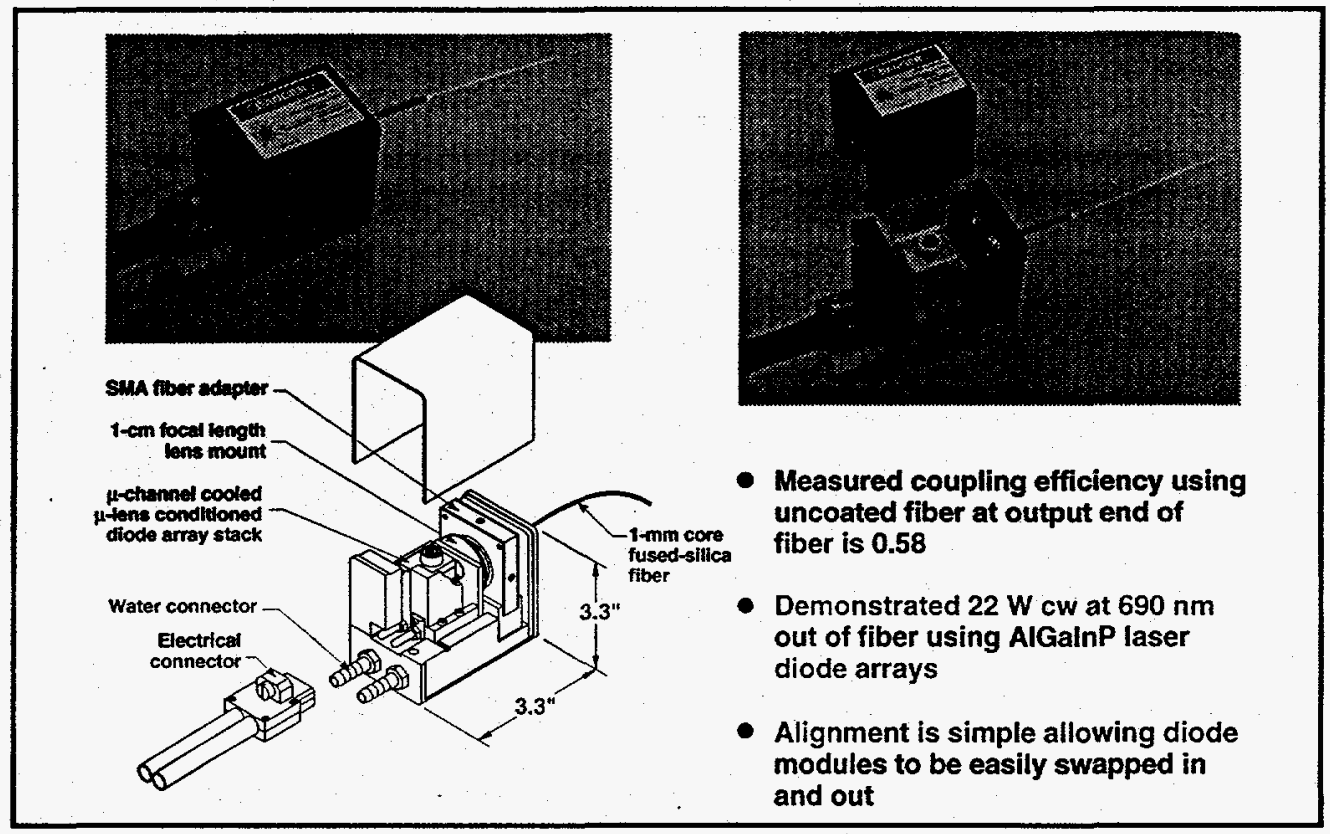

Figure 14. Photographs and drawing of LLNL diode-to-fiber coupling unit.

Yet another application of radiance conditioned laser diode arrays is as a high-intensity radiation source for surface heat treatment. Radiative heating using a laser source provides an attractive technique for performing the surface treatment of metallic parts. The primary reason for performing such surface heat treatments is to improve their hardness and resistance to wear. Laser diode arrays equipped with appropriate radiance conditioning, such as our microlenses, provide an efficient, reliable, and relatively inexpensive source of radiation suitable for performing such surface treatments. Typical industrial examples of where such direct-laser-diode heat-treating systems could be of use are found in the automotive world where it is desirable to harden localized portions of various automobile engine components. Particular examples of this would be automobile engine camshafts and valve seats. The photograph in Figure 15 shows a microlens-conditioned 10 module stack of diodes. The appropriate figure of merit for systems such as this which are intended to deliver high intensity radiation to a work piece is the optical radiance or the W/cm 2 -sr emitted by the device. Each module in the stack in Figure 15 holds a $1.5 \mathrm{~cm}$ long by 500 micron cavity length InGaAs laser diode bar emitting at $950 \mathrm{~nm}$. Operating such individual modules at $40 \mathrm{~W} \mathrm{CW}$ for several hundred hours and extrapolating their performance indicates lifetimes of greater than 3000 
hours until the package performance has degraded by $30 \%$. The CW irradiance measured at the face of the stack under these operating conditions is $400 \mathrm{~W} / 1.5 \mathrm{~cm}^{2}=267 \mathrm{~W} / \mathrm{cm}^{2}$. With the microlens conditioning which at present is $80 \%$ efficient in collecting and collimating the emitted diode radiation, the farfield radiation from an extended stack is contained in a angular region having FWHM of $20 \mathrm{mR}$ in the fast axis (conditioned) direction by $140 \mathrm{mR}$ in the slow axis direction. This leads to a stack radiance of

$$
R_{\text {stack }}=\frac{\eta_{\text {microlens }} \cdot P_{\text {stack }}^{c w}}{A_{\text {stack }} \cdot \Delta \Omega_{\text {stack }}}=\frac{0.8 \cdot 400 \mathrm{~W}}{1.5 \mathrm{~cm}^{2} \cdot 0.02 \cdot 0.14}=7.6 \cdot 10^{4} \frac{\mathrm{W}}{\mathrm{cm}^{2}-\mathrm{sr}}
$$

If this radiation were to be delivered by an optical system with an NA of 0.5 , so that the radiation was delivered within a solid angle of 1 steradian, the intensity at the work piece would be $76 \mathrm{~kW} / \mathrm{cm}^{2}$ (assuming no losses in the optical delivery system). LLNL is presently engaged in a collaborative effort to demonstrate such a system for a hardening application in which the output radiation of the microlens-conditioned stack is formatted using a simple set of crossed cylindrical lenses.

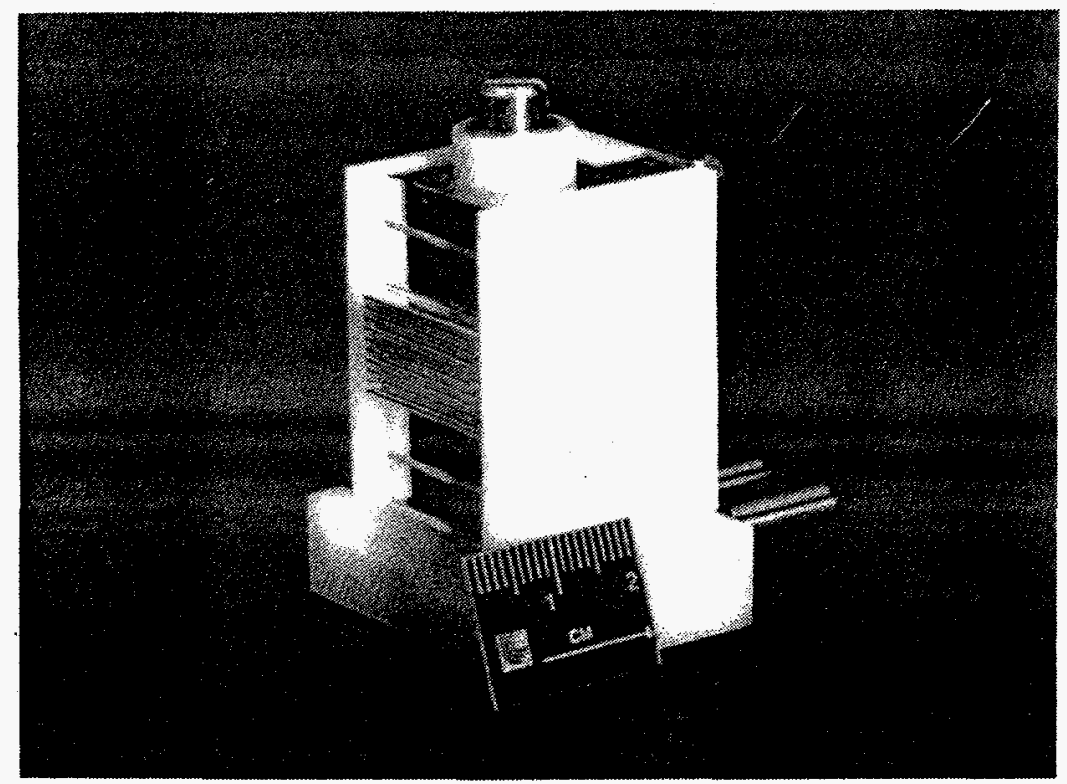

Figure 15. Photograph of 10 bar microlens-conditioned stack of laser diode arrays used in surface heat treating studies.

\section{CONCLUSION}

A modular, actively cooled laser diode array heatsink has been developed utilizing a silicon-based microchannel cooling technology that allows individual diode bars to be run at high average power and to be efficiently and inexpensively microlens-conditioned. The microlenses are manufactured using a simple and low cost shaped-fiber pulling technology developed by LLNL. The LLNL package was developed for use in pumping solid state lasers and enables the realization of very compact and lightweight DPSSL systems. The simplicity of the stacking procedure used to build up twodimensional apertures enhances the flexibility of the LLNL approach, as arbitrarily sized arrays can be constructed using the same basic technology. Furthermore, the array can be inexpensively maintained since a defective package can be easily replaced. Ease of manufacturability and limiting packaging costs have been key considerations in the development of the LLNL package. These points have been addressed by constructing the package from low cost silicon and glass, and by taking advantage of previously well developed silicon processing technologies in the package fabrication. The use of polished silicon wafers leads naturally to a low smile package which is essential if radiance conditioning (using cylindrical microlenses) is to be efficient. Using microlens-conditioned stacks of laser diode arrays in conjunction with the LLNLdeveloped lensing duct technology enables an inexpensive and commercially attractive diode end-pumping architecture that is simply and easily scaled in average power. This technology has been by employed by LLNL in a Nd:YLF laser oscillator, a wing pumped Cr:LiSAF laser, and a $2 \mu \mathrm{m}$ Tm:YAG laser. Future applications of this end-pumping technology will likely center on developing high average power DPSSL systems for both defense and industrial applications. Taking 
advantage of the high pump intensities that can be generated, ions such as $\mathrm{Yb}^{3+}$ with more favorable thermal power generation properties than $\mathrm{Nd}^{3+}$, can be exploited in high average power DPSSLs.

Because microlens-conditioned laser diode arrays provide a source radiance intermediate between that of unconditioned laser diodes at the low end and DPSSLs at the high end, there exists numerous applications in which such conditioned arrays are useful on a stand-alone basis. One example of where the intermediate radiance of microlensconditioned stacks of laser diode arrays is finding application is in direct diode-to-fiber coupling. Such systems have application in both the medical and material processing fields. Another example is the use of direct microlens-conditioned diode stacks for surface treatment applications. Surface hardening and other heat treating applications continue to expand for such systems.

There has recently developed outside interest in commercializing the technology presented within. One of the important advantages of our technology verses that already developed and available commercially is the ability to engage such a broad spectrum of high average power diode laser applications using a simple base package design that allows the simple integration of radiance-conditioning cylindrical microlenses.

\section{ACKNOWLEDGMENTS}

We thank W. Krupke, G. F. Albrecht, S. B. Sutton, B. Comaskey, and E. V. George of Lawrence Livermore National Laboratory for the many useful discussions and stimulating arguments that occurred during the course of this work. We also thank Jim Snyder of Blue Sky Research who developed the shaped microlens technology while at Lawrence Livermore National Laboratory. Additionally, for their technical contributions to this work, the authors wish to express their appreciation and thanks to V. Sperry, D. Ciarlo, T. Rodriguez, L. DiMercurio, E. Utterback, D. VanLue, C. Reinhardt, and P. Reichert. Finally, for their continued encouragement and support of the activities described within, we wish to express our appreciation and thanks to H. T. Powell and E. M. Campbell of Lawrence Livermore National Laboratory.

This work was performed under the auspices of the U.S. Department of Energy by Lawrence Livermore National Laboratory under contract W-7405-Eng-48.

\section{REFERENCES}

1. R. Beach, W. Benett, B. Freitas, D. Mundinger, B. Comaskey, R. Solarz, and M. Emanuel, "Modular microchannel cooled heatsinks for high average power laser diode arrays," IEEE J. Quantum Electron., Vol. 28, p. 966, 1992.

2. W. J. Benett, B. L. Freitas, D. Ciarlo, R. Beach, S. Sutton, M. Emanuel, and R. Solarz, "Microchannel cooled heatsinks for high average power laser diode arrays," SPIE, Vol. 1865, p. 144, 1993.

3. R. J. Beach, M. A. Emanuel, W. J. Benett, B. L. Freitas, D. Ciarlo, N. W. Carlson, S. B. Sutton, J. A. Skidmore, and R.W. Solarz, "Improved performance of high average power semiconductor arrays for application in diode pumped solid state lasers," SPIE Proceedings, Vol. 2148, pp. 25-27, Jan. 1994.

4. T. J. Bland, R. E. Niggemann, and M. B. Parekh, "A Compact High Intensity Cooler (CHIC)," SAE Technical Paper Series, Thirteenth Intersociety Conf. on Environmental Systems, San Francisco, CA, July 11-14, 1983.

5. D. Tuckerman and R. Pease, "High-performance heatsinking for VLSI," IEEE Electron. Device Letters, Vol. EDL-2, p. $126,1981$.

6. D. Tuckerman, "Heat-transfer microstructures for integrated circuits," Ph.D. dissertation, Stanford University, Stanford, CA, 1984.

7. $\quad$ R. J. Phillips, "Microchannel heat sinks," The Lincoln Laboratory Journal, Vol, 1, No. 1, p. 31, 1988.

8. G. Wallis and D. Pomerantz, "Field assisted glass-metal sealing," J. Appl. Phys., Vol. 40, p. 3946, 1969.

9. J.J . Snyder, P. Riechert, and T. Baer, "Fast diffraction-limited cylindrical microlenses," Appl. Opt., Vol. 30, p. 2743, 1991.

10. J. J. Snyder, "Cylindrical micro-optics," SPIE Proceedings, Vol. 1992, pp. 14-16, July 1993.

11. G. L. Harnagel, M. Vakili, K. R. Anderson, D. P. Worland, J. G. Endriz, and D. R. Scifres, "High-duty cycle, highpower two-dimensional laser diode arrays," Electronic Letters, Vol. 29, No. 11, p. 1008, 1993.

12. R. Beach, P. Reichert, W. Benett, S. Mitchell, S. Velsko, J. Davin, and R. Solarz, "Scalable diode-end-pumping technology applied to a 100-mJ Q-switched Nd3+:YLF laser oscillator," Optics Letters, Vol. 18, p. 1326, 1993.

13. M. D. Perry, S. A. Payne, T. Ditmire, R. Beach, G. J. Quarles, W. Ignatuk, R. Olson, and J. Weston, "Better materials trigger Cr:LiSAF laser development," Laser Focus World, p. 85, Sept. 1993.

14. M. D. Jones, J. R. Rotge, and D. M. Gianardi, Jr., "Compact robust fiber-optic coupled diode laser array module for solid-state laser pumping and beacon applications," SPIE Proceedings, Vol. 2148, pp. 25-27, Jan. 1994. 